\title{
Controle da leishmaniose visceral em meio urbano: estudo de intervenção randomizado fatorial
}

\author{
Control of visceral leishmaniasis in urban areas: \\ randomized factorial intervention trial
}

\author{
Carlos Henrique Nery Costa ${ }^{1}$, Conceição Maria M. Tapety ${ }^{1}$ \\ e Guilherme L. Werneck ${ }^{2,3}$
}

\begin{abstract}
RESUMO
O objetivo deste trabalho foi avaliar a efetividade do controle vetorial e da eliminação de cães infectados na incidência de infecção por Leishmania chagasi. Um estudo de intervenção comunitário foi realizado em Teresina entre 1995 e 1996. A área foi dividida em 34 lotes alocados aleatoriamente a 4 tipos de intervenção: 1) borrifação intradomiciliar e de anexos residenciais; 2) borrifação intradomiciliar e eliminação de cães infectados; 3) combinação de borrifação intradomiciliar e de anexos e eliminação canina; 4) apenas borrifação intradomiciliar. Em comparação com lotes que receberam apenas borrifação intradomiciliar, a eliminação canina diminui em $80 \%$ a incidência de infecção. A borrifação de anexos, associada ou não à eliminação canina, não apresentou efeito significativo. A proteção oferecida pela remoção de cães infectados sugere que esta estratégia pode reduzir o pool de fontes de infecção para flebotomíneos.
\end{abstract}

Palavras-chaves: Leishmaniose visceral. Controle. Epidemiologia. Estudo de intervenção.

\begin{abstract}
The objective of this study was to evaluate the effect of vector control and elimination of infected dogs on the incidence of infection with Leishmania chagasi. A randomized community intervention trial was carried out in Teresina between 1995 and 1996. The area was divided in 34 blocks randomically allocated to 4 types of intervention: 1) spraying houses and animal pens with insecticide; 2) spraying houses and eliminating infected dogs; 3) combination of spraying houses and animal pens, and eliminating infected dogs, and 4) only spraying houses. In comparison to blocks receiving only household spraying, culling dogs decreased in $80 \%$ the incidence of infection. Spraying animal pens, associated or not with culling dogs, showed no significant effect. The protection offered by culling dogs suggests that this strategy might reduce the source of infection for the vector.
\end{abstract}

Key-words: Visceral leishmaniasis. Control. Epidemiology. Intervention study.

A leishmaniose visceral (LV) é uma doença emergente em diferentes áreas urbanas brasileiras. Transformações ambientais associadas a movimentos migratórios e ao processo de urbanização podem explicar, em parte, porque a LV, originalmente uma doença restrita às áreas rurais, passou a ocorrer de forma endêmica e epidêmica em grandes cidades do nordeste brasileiro ${ }^{514}$. Este processo desordenado de ocupação urbana resultou em condições precárias de vida e destruição ambiental, fatores que também podem ter influenciado a emergência da doença no meio urbano. Por um lado, o vetor Lutzomyia longipalpis se adapta facilmente às condições peridomésticas de áreas depauperadas, explorando o acúmulo de matéria orgânica gerada por animais domésticos e más-condições sanitárias ${ }^{1}$. Por outro, cães abandonados vagando na periferia da cidade podem se infectar quando entram em contato direto com reservatórios selvagens e, ao retornarem para o interior da cidade, servirem de amplificadores da infecção para outros cães e humanos.

Fundamentado nesta concepção de transmissão, o programa brasileiro de controle da leishmaniose visceral baseia sua estratégia em três medidas, sendo que a primeira delas tem caráter eminentemente curativo: 1) detecção e tratamento de

\footnotetext{
1. Laboratório de Leishmanioses do Instituto de Doenças Tropicais Natan Portella, Teresina, PI. 2. Departamento de Epidemiologia do Instituto de Medicina Social da Universidade do Estado do Rio de Janeiro, Rio de Janeiro, RJ. 3. Instituto de Estudos em Saúde Coletiva da Universidade Federal do Rio de Janeiro, Rio de Janeiro, RJ. Apoio: CNPq Processo 467183-007; CNPq Processo 305018/2002-7

Endereço para correspondência: Dr. Guilherme L. Werneck. Dept ${ }^{\circ}$ de Epidemiologia/IMS/UERJ. R. São Francisco Xavier 524/7ํandar, Bloco D, Maracanã, 20559-900 Rio de Janeiro, RJ.

Tel: $55212587-7303$ ramal 22

e-mail: gwerneck@nesc.ufrj.br

Recebido em: 10/02/2005

Aceito em: 11/07/2007
} 
casos humanos, 2) controle dos reservatórios domésticos, e 3) controle de vetores ${ }^{12}$. 0 que sustenta a utilização do controle vetorial e de reservatórios como estratégias de intervenção sobre a LV é a conjectura de que a incidência de infecção em humanos é diretamente relacionada ao número de cães infectantes e a fatores entomológicos ${ }^{49}$. Entretanto, após anos de investimento humano e financeiro, nota-se que os resultados foram desalentadores e a LV avança para se tornar mais uma mazela do cotidiano urbano brasileiro. Uma razão fundamental para a inefetividade destas estratégias é a necessidade de um sistema de vigilância permanente, com utilização extensiva de recursos humanos e financeiros, o que limita sua sustentabilidade e cobertura. Outros fatores, como a possibilidade de transmissão por cães assintomáticos, má-qualidade dos procedimentos diagnósticos, e a própria dinâmica populacional canina, com a rápida aquisição de infecção pelos novos cães introduzidos em substituição aos cães eliminados, podem influir na inefetividade da eliminação canina $^{679}$.

Estudos enfocando a estratégia de eliminação canina têm oferecido resultados conflitantes ${ }^{3}$, pelo menos quando utilizada separadamente do controle vetorial. Por outro lado, experiências têm demonstrado a efetividade do controle vetorial em diversas situações ${ }^{25}{ }^{13}$. Neste contexto, merece destaque um estudo realizado em Porteirinha, norte de Minas Gerais, que identificou uma redução na incidência de leishmaniose canina e humana, após a retirada sistemática de cães soropositivos, no mesmo momento em que o controle vetorial também foi implementado $0^{11}$. Entretanto, ainda são poucos os estudos brasileiros especificamente delineados para testar conjuntamente a efetividade do controle vetorial e da eliminação canina na redução da incidência de infecção humana. Também, do ponto de vista metodológico, seria importante superar algumas limitações comuns a estes estudos, quais sejam, o uso de poucas áreas de comparação e a variabilidade nas taxas de transmissão basal da infecção, o que leva a uma inerente falta de comparabilidade entre as áreas. Este trabalho tem como objetivo avaliar a efetividade do controle vetorial e da eliminação de cães infectados na incidência de infecção por Leishmania chagasi, em um cenário urbano com nível de transmissão homogênea.

\section{MATERIAL E MÉTODOS}

Este estudo de intervenção comunitário com desenho fatorial foi realizado em Teresina entre 1995 e 1996 no bairro Itararé, onde a transmissão de Leishmania chagasi é ativa. 0 Itararé é o bairro mais populoso de Teresina, contando, em 1996, com quase 45 mil habitantes. Apresentando área de cerca de 3,5km², o bairro está localizado na região sudeste da cidade. Durante a década de 1990, cerca de 109 casos de leishmaniose visceral foram registrados entre moradores do bairro, o que corresponde a uma incidência média anual de cerca de 2,5 casos por 100.000 habitantes, posicionando o bairro próximo da incidência média de LV em Teresina.
Para fins deste estudo, a parte central do bairro foi subdividida em 34 lotes de aproximadamente $200 \mathrm{~m} \times 200 \mathrm{~m}$ (Figura 1). 0 s lotes foram alocados aleatoriamente a 4 tipos de intervenção: A) borrifação intradomiciliar e de anexos residenciais; B) borrifação intradomiciliar e eliminação de cães infectados; C) combinação de borrifação intradomiciliar e de anexos residenciais e eliminação canina $\mathrm{eD}$ ) apenas borrifação intradomiciliar. As áreas em que se executou apenas borrifação intradomiciliar foram consideradas áreas de não-intervenção. Apenas as residências localizadas nos $100 \mathrm{~m} \times 100 \mathrm{~m}$ interiores de cada lote foram selecionadas para 0 estudo, de forma a criar um buffer para minimizar a possibilidade de contaminação cruzada das intervenções entre as áreas.

Os procedimentos de borrifação envolveram a aplicação de inseticida piretróide nas paredes internas de todos os domicílios (borrifação intradomiciliar) e de anexos residenciais apenas nas residências de lotes sorteados para borrifação extradomiciliar. A técnica de aspersão envolveu borrifação de $100 \%$ das paredes numa altura de $3 \mathrm{~m}$, obedecendo à sequiência dos ponteiros do relógio, removendo todos os objetos da parede antes da aplicação.

Em relação à eliminação canina nas áreas sorteadas para receberem esta intervenção, procedeu-se à coleta de amostra de sangue em papel de filtro dos cães encontrados na região através de um estilete aplicado na ponta da orelha. A técnica utilizada para o diagnóstico foi a reação de imunofluorescência indireta (RIFI). Os cães considerados positivos (RIFI $\geq 1: 40$ ) foram eliminados de acordo com técnicas preconizadas.

Trezentos e sessenta e sete indivíduos distribuídos pelos 34 lotes consentiram em fornecer amostras de sangue para a realização de exames sorológicos para identificação de anticorpos à Leishmania chagasi. A técnica de ELISA foi utilizada para a detecção de anticorpos para Leishmania chagasi de acordo com métodos previamente descritos ${ }^{10} \mathrm{com}$ antígenos derivados de cepas de Leishmania chagasi (MHOM/BR/74/PP75). Duas visitas semestrais foram agendadas para realização de nova coleta de sangue e avaliação da soroconversão.

Análise estatística foi realizada através de regressão logística multivariada, estimando-se a razão de chances odds ratio (OR) como medida de associação entre as variáveis e seus respectivos intervalos de 95\% de confiança (IC 95\%), controlando para os efeitos de idade e sexo. Especificou-se um modelo que considerou explicitamente o efeito de agregação dos indivíduos em lotes (efeito de cluster) e utilizou-se métodos de estimação robusta da variância. A análise de dados foi realizada no aplicativo STATA 6.0 .

\section{RESULTADOS}

Na Tabela 1, observa-se que de um total de 367 indivíduos selecionados inicialmente, 154 tiveram exame positivo (prevalência de infecção na linha de base de $42 \%$ ). As prevalências de soropositividade na linha de base foram similares nas áreas de intervenção, mas significativamente mais baixa na área de nãointervenção (apenas borrifação intradomiciliar). 


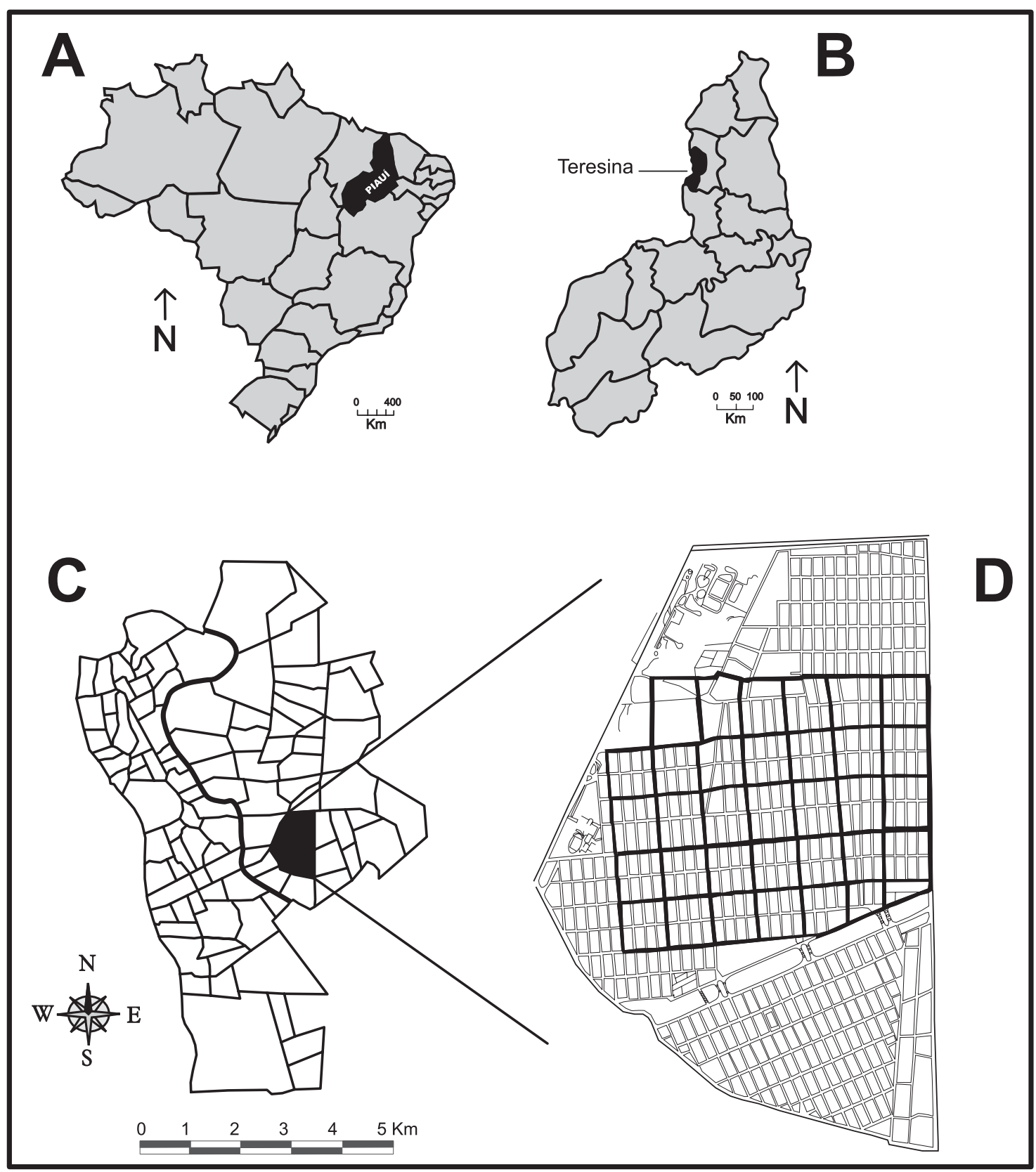

Figura 1 - A) Estado do Piauí, Brasil. B) Cidade de Teresina, Estado do Piauí. C) Divisão de administrativa por bairros, Teresina. D) Bairro do Itararé.

Tabela 1 - Prevalências, incidências, odds ratios e intervalos de 95\% de confiança (IC 95\%) para a associação entre diferentes intervenções e infecção por Leishmania chagasi, Teresina, 1995-1996.

\begin{tabular}{lcccr}
\hline Área & Prevalência (\%) & Incidência (\%) & OR* & IC 95\%* \\
\hline A & 46,0 & 38,7 & 0,64 & $0,19-2,18$ \\
B & 46,0 & 16,1 & 0,20 & $0,04-0,89$ \\
C & 40,0 & 37,9 & 0,69 & $0,27-1,76$ \\
D & 31,3 & 48,3 & 1,00 & - \\
\hline Total & 42,0 & 35,0 & - & -
\end{tabular}

* ajustado para sexo e idade OR: odds ratios IC 95\%: intervalos de 95\% de confiança

A: borrifação intradomiciliar e de anexo residenciais B: borrifação intradomiciliar e eliminação de cães infectados. C: combinação de borrifação intradomiciliar e de anexos residenciais e eliminação canina e D: apenas borrifação intradomiciliar (não intervenção).
Dos 213 soronegativos inicialmente selecionados para 0 seguimento, apenas 120 (56\%) tiveram sangue coletado em pelo menos mais uma ocasião, com intervalo mínimo de 6 meses, para avaliação da soroconversão. A Tabela 1 mostra que a incidência acumulada de infecção no período de observação (entre 6 meses e 1 ano) foi de 35\% (42/120).

Resultados de análise multivariada mostraram que, em comparação com os lotes que receberam apenas borrifação intradomiciliar, a eliminação canina diminui em $80 \%$ a incidência de infecção. Por outro lado, a borrifação de anexos adicionalmente a borrifação intradomiciliar sem eliminação de cães infectados ou com eliminação de cães não esteve significantemente associada à redução da soroconversão (Tabela 1). 


\section{DISCUSSÃo}

Os resultados deste estudo apontam para um efeito protetor da eliminação de cães infectados na incidência de infecção pela Leishmania chagasi adicionalmente ao potencial efeito protetor propiciado pela borrifação intradomiciliar. Entretanto, a borrifação de anexos, associada ou não à eliminação canina, não adicionou efeito protetor significativo ao induzido pela borrifação intradomiciliar.

0 efeito protetor da eliminação de cães infectados encontra fundamento no conhecimento atual sobre a transmissão desta infecção. A remoção de cães infectados estaria reduzindo o pool de fontes de infecção para flebotomíneos, limitando a capacidade destes vetores em transmitir o parasito para humanos.

A ausência de efeito protetor oferecido pela borrifação extradomiciliar pode decorrer de vários motivos. Primeiro, é necessário salientar que este estudo avalia o efeito adicional da borrifação extradomiciliar, já que todos os domicílios foram submetidos à borrifação intradomiciliar. Sendo assim, é possível que o efeito potencial máximo a ser obtido através do controle vetorial já tivesse sido alcançado pela borrifação intradomiciliar, provocando um esgotamento do potencial protetor das estratégias suplementares de borrifação. Segundo é possível que a borrifação extradomiciliar, ao mesmo tempo em que diminui a população e longevidade dos flebotomíneos, esteja provocando também maior dispersão dos flebotomíneos restantes, que passariam a circular em outras regiões do peridomicílio aumentando seus contatos com humanos. Neste caso, o potencial efeito protetor decorrente da diminuição da população e longevidade dos insetos, estaria sendo compensado pelo aumento da taxa de picadas em humanos. Não se pode descartar também a possibilidade de problemas operacionais na aplicação do inseticida, assim como a falta de eficácia do próprio inseticida empregado, seja devido à diluição errônea, potencial inseticida insuficiente, expiração de prazo de validade, ou resistência. Infelizmente, nenhum controle de qualidade, além daquele realizado pelo próprio serviço de controle de zoonoses da região, pode ser realizado para avaliar estas hipóteses.

0 resultado mais intrigante, entretanto, é a ausência de efeito da eliminação canina quando é feito concomitantemente a borrifação extradomiciliar. Ainda que as explicações apresentadas anteriormente possam justificar a não efetividade da borrifação extradomiciliar adicionalmente à borrifação intradomiciliar, seria esperado algum efeito devido à eliminação canina, já que esta se mostrou efetiva quando avaliada isoladamente. Uma possível explicação seria equivalente à segunda opção delineada anteriormente, o efeito da remoção de cães infectados estaria sendo compensado pelo efeito de dispersão induzido pela borrifação de anexos domiciliares.

Apesar das vantagens do desenho de estudo empregado, com utilização de estratégias de alocação aleatória de intervenções e do delineamento fatorial, que permite avaliar a interação entre as duas medidas de controle, este estudo apresenta várias limitações que precisam ser cuidadosamente consideradas para garantir uma interpretação isenta dos resultados encontrados.
Primeiro, a medida de incidência de infecção utilizada não parece ser a mais apropriada, melhor teria sido obter também informações sobre intradermorreação com leishmanina. Segundo, a definição de grupo de não-intervenção não é a mais adequada, já que este grupo sofreu algum tipo de intervenção (borrifação intradomiciliar). Terceiro, as perdas de seguimento alcançaram quase $50 \%$ da amostra, o que além de limitar o poder estatístico do estudo, também pode ter induzido a vieses nas estimativas de efeito. Por fim, a variação na intensidade da infecção entre as áreas de intervenção, conforme verificado pela prevalência diferenciada da infecção nas diferentes áreas de intervenção, pode ter sido responsável por parte dos efeitos (ou ausência de efeitos) observados.

Apesar destas importantes limitações, este estudo tem relevância para a construção do conhecimento sobre a efetividade das intervenções em leishmaniose visceral, principalmente por ser um dos primeiros especificamente delineados para testar conjuntamente a efetividade do controle vetorial e da eliminação canina na redução da incidência de infecção humana, através de um desenho epidemiológico robusto com uso de um número razoável de áreas de comparação. Entretanto, os resultados aqui apresentados devem ser recebidos com cautela, e novos estudos de intervenção randomizados com desenho fatorial devem ser realizados em áreas com níveis variados de transmissão e buscando minimizar os problemas enfrentados na condução deste estudo.

\section{REFERÊNCIAS}

1. Aguiar GM, Medeiros WM, De Marco TS, Santos SC, Gambardella S. Ecologia dos flebotomíneos da Serra do Mar, Itaguaí, Estado do Rio de Janeiro, Brasil. I - A fauna flebotomínica e prevalência pelo local e tipo de captura (Diptera, Psychodidae, Phlebotominae). Cadernos de Saúde Pública 12:195-206, 1996.

2. Alencar J. Profilaxia do calazar no Ceará, Brasil. Revista do Instituto de Medicina Tropical de São Paulo 3:175-180, 1961.

3. Ashford DA, David JR, Freire M, David R, Sherlock I, Eulalio MC, Sampaio DP, Badaró R. Studies on control of visceral leishmaniasis: impact of dog control on canine and human visceral leishmaniasis in Jacobina, Bahia, Brazil. The American Journal of Tropical Medicine and Hygiene 59:53-57, 1998.

4. Burattini MN, Coutinho FAB, Lopez LF, Massad E. Modelling the Dynamics of Leishmaniasis Considering Human, Animal Host and Vector Populations. Journal of Biological Systems 6:337-356, 1998.

5. Costa CHN, Pereira HF, Araújo MV. Epidemia de leishmaniose visceral no estado do Piauí, Brasil, 1980-1986. Revista de Saúde Pública 24:361-372, 1990.

6. Costa CHN, Vieira JBF. Mudanças no controle da leishmaniose visceral no Brasil. Revista da Sociedade Brasileira de Medicina Tropical 34:223-228, 2001.

7. Courtenay O, Quinnell RJ, Garcez LM, Shaw JJ, Dye C. Infectiousness in a cohort of Brazilian dogs: why culling fails to control visceral leishmaniasis in areas of high transmission. Journal of Infectious Diseases 186:1314-1320, 2002.

8. Dietze R, Barros GB, Teixeira L, Harris J, Michelson K, Falqueto A, Corey R. Effect of eliminating seropositive canines on the transmission of visceral leishmaniasis in Brazil. Clinical Infectious Diseases 25:1240-1242, 1997.

9. Dye C. The logic of Visceral Leishmaniasis control. The American Journal of Tropical Medicine \& Hygiene 55:125-130, 1996.

10. Evans TG, Krug EC, Wilson ME, Vasconcelos AW, Alencar JE, Pearson RD. Evaluation of antibodies responses in American visceral leishmaniasis by ELISA and immunoblot. Memórias do Instituto Oswaldo Cruz 84:157-166, 1989.

11. França-Silva JC. Distribuição Espacial e Temporal da Leishmaniose Visceral Canina em Relação à Densidade Vetorial e ao Controle de Cães Infectados em 
Porteirinha-Minas Gerais (1998-2002). Tese de Doutorado, Universidade Federal de Minas Gerais, Belo Horizonte, MG, 2003.

12. Fundação Nacional de Saúde. Controle, diagnóstico e tratamento da leishmaniose visceral (Calazar): Normas técnicas. Ministério da Saúde. Brasília, 2003.
13. Gupta S. Return of kala-azar. Journal of Indian Medical Association 65:89-90, 1991

14. Mendes WS, Silva AAM, Trovão JR, Silva AR, Costa JML. Expansão espacial da leishmaniose visceral americana em São Luis, Maranhão, Brasil. Revista da Sociedade Brasileira de Medicina Tropical 35: 227-231, 2002. 